\title{
The relationship between legal coercion and dropout from substance abuse treatment Brian Perron
}

\author{
Address: Washington University, One Brookings Drive, St. Louis, Missouri 63130, USA \\ from WPA Thematic Conference. Coercive Treatment in Psychiatry: A Comprehensive Review \\ Dresden, Germany. 6-8 June 2007 \\ Published: 19 December 2007 \\ BMC Psychiatry 2007, 7(SuppI I):PI4 doi:10.II86/I47I-244X-7-SI-PI4
}

This abstract is available from: http://www.biomedcentral.com/l47I-244X/7/SI/PI4

(C) 2007 Perron; licensee BioMed Central Ltd.

\section{Background}

Legal coercion is a popular method for leveraging substance abuse treatment among people who would otherwise not participate voluntarily. Although it is effective for getting people into treatment, the results have been mixed as to whether people who are legally coerced stay in treatment longer and have better treatment outcomes. Many studies have relied on small, clinic-based surveys that are not generalizable to the broader treatment system. This study attempts to fill this gap in knowledge by comparing dropout among legally coerced and voluntary clients using a national survey.

\section{Methods}

This study used data from the National Treatment Improvement Evaluation Study (NTIES). The NTIES was a prospective study of the impact of treatment programs on persons in publicly funded substance abuse treatment program. Subjects for this study were from outpatient non-methadone and non-correctional settings $(\mathrm{N}=$ 1,439 ) from 31 different treatment programs. The primary analytic strategy was a type of survival analysis called Cox proportional hazards regression.

\section{Results}

Approximately $77 \%$ of the entire sample failed to complete treatment. A chi-square analysis revealed that the rate of dropout among persons legally coerced to treatment was no different than persons who attended treatment voluntarily. A Cox proportional hazards regression showed that legal coercion reduced the risk of dropout by approximately $7 \%$. The strongest predictors of dropout were being male and substance use severity.

\section{Conclusion}

Legal coercion may be an effective way of getting persons into treatment. However, the results suggest that it leads to only a slight reduction in the risk of treatment dropout. This raises questions regarding the overall effectiveness of this mechanism to leverage persons who are resistant to entering treatment voluntarily. Further efforts are needed to develop non-coercive methods to effectively engage persons who are resistant to treatment. 